\title{
MULTIPLIERS OF MULTIDIMENSIONAL FOURIER ALGEBRAS
}

\author{
I. G. TODOROV AND L. TUROWSKA
}

\begin{abstract}
Let $G$ be a locally compact $\sigma$-compact group. Motivated by an earlier notion for discrete groups due to Effros and Ruan, we introduce the multidimensional Fourier algebra $A^{n}(G)$ of $G$. We characterise the completely bounded multidimensional multipliers associated with $A^{n}(G)$ in several equivalent ways. In particular, we establish a completely isometric embedding of the space of all $n$-dimensional completely bounded multipliers into the space of all Schur multipliers on $G^{n+1}$ with respect to the (left) Haar measure. We show that in the case $G$ is amenable the space of completely bounded multidimensional multipliers coincides with the multidimensional Fourier-Stieltjes algebra of $G$ introduced by Ylinen. We extend some well-known results for abelian groups to the multidimensional setting.
\end{abstract}

Mathematics subject classification (2010): Primary 47B49; Secondary 47L60, 43A22.

Keywords and phrases: Fourier algebra, multiplier, Schur multiplier, multidimensional.

\section{REFERENCES}

[1] D. P. Blecher And C. Le Merdy, Operator algebras and their modules - an operator space approach, Oxford University Press, 2004.

[2] D. P. Blecher And R. Smith, The dual of the Haagerup tensor product, J. London Math. Soc. (2), 45 (1992), 126-144.

[3] M. BOZEJKo AND G. FendleR, Herz-Schur multipliers and completely bounded multipliers of the Fourier algebra of a locally compact group, Colloquium Math., 63 (1992), 311-313.

[4] J. DE CANNiÈRE AND U. HAAGERUP, Multipliers of the Fourier algebras of some simple Lie groups and their discrete subgroups, Amer. J. Math., 107, 2 (1985), 455-500.

[5] C. Cecchini, Operators on $\mathrm{VN}(G)$ commuting with $A(G)$, Colloquium Math., 43 (1980), 137-142.

[6] E. ChristensEn AND A. M. Sinclair, Representations of completely bounded multilinear operators, J. Funct. Anal., 72 (1987), 151-181.

[7] E. G. EFFROS AND ZH.-J. RUAN, Multivariable multipliers for groups and their operator algebras, Proceedings of Symposia in Pure Mathematics, 51 (1990), Part 1, 197-218.

[8] E. G. EFFros AND ZH.-J. RuAn, Operator Spaces, London Mathematical Society Monographs. New Series, 23. The Clarendon Press, Oxford University Press, New York, 2000. xvi+363 pp.

[9] E. G. EFFROS AND ZH.-J. RUAN, Operator spaces tensor products and Hopf convolution algebras, J. Operator Theory, 50 (2003), 131-156.

[10] P. EYMARD, L'alg èbre de Fourier d'un groupe localement compact, Bulletin de la S.M.F., 92 (1964), $181-236$.

[11] J. E. Gilbert, T. Ito AND B.M. Schreiber, Bimeasure algebras on locally compact groups, J. Funct. Anal., 64 (1985), 134-162.

[12] C. Graham And B. M. Schreiber, Bimeasure algebras on LCA groups, Pacific J. Math., 115, 1 (1984), 91-127.

[13] A. GRothendieck, Resume de la theorie metrique des produits tensoriels topologiques, Boll. Soc. Mat. Sao-Paulo, 8 (1956), 1-79.

[14] P. Joliss AINT, A characterisation of completely bounded multipliers of Fourier algebras, Colloquium Math., 63 (1992), 311-313.

[15] K. Juschenko, I. G. TOdorov And L. Turows Ka, Multidimensional operator multipliers, Trans. Amer. Math. Soc., 361, 9 (2009), 4683-4720.

[16] V. PAulsen, Completely bounded maps and operator algebras, Cambridge University Press, 2002. 
[17] V. V. PELLER, Hankel operators in the perturbation theory of unitary and selfadjoint operators, Funktsional. Anal. i Prilozhen., 19, 2 (1985), 37-51, 96.

[18] G. PISIER, Similarity problems and completely bounded maps, Second, expanded edition. Includes the solution to "The Halmos problem". Lecture Notes in Mathematics, 1618. Springer-Verlag, Berlin, 2001. viii+198 pp.

[19] G. PISIER, Introduction to Operator Space Theory, Cambridge University Press, 2003.

[20] W. RUdin, Fourier analysis on groups, Interscience Tracts in Pure and Applied Mathematics, No. 12, Interscience Publishers (a division of John Wiley and Sons), New York-London $1962 \mathrm{ix}+285 \mathrm{pp}$.

[21] N. Spronk, Measurable Schur multipliers and completely bounded multipliers of the Fourier algebras, Proc. London Math. Soc. (3), 89, 1 (2004), 161-192.

[22] K. YLINEN, Non-commuattive Fourier transforms of bounded bilinear forms and completely bounded multilinear operators, J. Funct. Anal., 79 (1988), 144-165.

[23] G. Zhao And B.M. Schreiber, Algebras of multilinear forms on groups, Contemp. Math., 189 (1995), 497-511. 